\title{
Flavorful leptoquarks at hadron colliders
}

\author{
Gudrun Hiller* and Dennis Loose ${ }^{\dagger}$ \\ Fakultät Physik, TU Dortmund, Otto-Hahn-Str.4, D-44221 Dortmund, Germany \\ Ivan Nišandžićł \\ Institut für Theoretische Teilchenphysik, Karlsruher Institut für Technologie, \\ D-76128 Karlsruhe, Germany
}

(Received 10 February 2018; published 3 April 2018)

\begin{abstract}
$B$-physics data and flavor symmetries suggest that leptoquarks can have masses as low as a few $\mathcal{O}(\mathrm{TeV})$, predominantly decay to third generation quarks, and highlight $p p \rightarrow b \mu \mu$ signatures from single production and $p p \rightarrow b b \mu \mu$ from pair production. Abandoning flavor symmetries could allow for inverted quark hierarchies and cause sizable $p p \rightarrow j \mu \mu$ and $j j \mu \mu$ cross sections, induced by second generation couplings. Final states with leptons other than muons including lepton flavor violation (LFV) ones can also arise. The corresponding couplings can also be probed by precision studies of the $B \rightarrow\left(X_{s}, K^{*}, \phi\right) e e$ distribution and LFV searches in $B$-decays. We demonstrate sensitivity in single leptoquark production for the large hadron collider (LHC) and extrapolate to the high luminosity LHC. Exploration of the bulk of the parameter space requires a hadron collider beyond the reach of the LHC, with $b$-identification capabilities.
\end{abstract}

DOI: 10.1103/PhysRevD.97.075004

\section{INTRODUCTION}

Leptoquarks generically couple differently to different generations of quarks and leptons. Present hints of nonuniversality between electrons and muons in the rare $B$-decay observables $R_{K}, R_{K^{*}}$ [1] by the LHCb collaboration [2,3] are indeed naturally explained by tree-level exchange of leptoquarks [4-16]. Combining $R_{K}$ with $R_{K^{*}}$ allows one to diagnose the chirality of the participating $|\Delta b|=|\Delta s|=1$ currents [17]. Current data favor leptoquarks that couple to quark and lepton doublets, e.g., [18-23], implying couplings to both $b$ - and $t$-quarks and charged leptons and neutrinos. The corresponding leptoquark representations are the scalar $S U(2)_{L}$-triplet $S_{3}$ and two vectors, a singlet $V_{1}$ and a triplet, $V_{3}$. Importantly, the mass scale of the leptoquarks is model-independently limited by multi- $O(10) \mathrm{TeV}$ and in viable flavor models in the $\mathrm{TeV}$ range, suggesting dedicated searches at the large hadron collider (LHC) and beyond [24].

Search results for leptoquarks are available for fixed branching fractions into a given lepton species. For instance, mass limits for leptoquarks decaying $50 \%$ to

\footnotetext{
*ghiller@physik.uni-dortmund.de dennis.loose@udo.edu

ivan.nisandzic@kit.edu
}

Published by the American Physical Society under the terms of the Creative Commons Attribution 4.0 International license. Further distribution of this work must maintain attribution to the author(s) and the published article's title, journal citation, and DOI. Funded by SCOAP ${ }^{3}$. electrons (muons) plus jet and the other $50 \%$ to neutrinos and a jet are $900 \mathrm{GeV}(850 \mathrm{GeV})$ [25], with similar results reported in $[26,27]$, all obtained from pair production. The limits improve to $1100 \mathrm{GeV}$ [26] (1080 GeV [27]) for $100 \%$ decay to electrons (muons) plus jet. Bounds from single production in jet $e e(1755 \mathrm{GeV})$ and jet $\mu \mu$ $(660 \mathrm{GeV})$ [28] assume 100\% decays to charged leptons and coupling equal to one. As we will show, rare $B$-decay data suggest to look for leptoquarks with dominant decays to $b \ell, \ell=e, \mu$. To date, no corresponding leptoquark search has been performed at the LHC. On the other hand, derived limits from other searches such as supersymmetry resulting in analogous signatures as the leptoquark ones are $1.5 \mathrm{TeV}\left(S_{3}\right)$ and $1.8 \mathrm{TeV}\left(V_{1,3}\right)$ for $b e$ and $1.4 \mathrm{TeV}\left(S_{3}\right)$ and $1.7 \mathrm{TeV}\left(V_{1,3}\right)$ for $b \mu$ [29]. Limits on $b \nu, t \ell$, and $t \nu$ are not stronger.

The aim of this study is to work out collider signatures of leptoquark scenarios that take into account flavor structure and $B$-physics data. We focus on single production, which is directly driven by the leptoquark couplings to quarks and leptons and results in signatures with a quark and two leptons. Flavor physics provides directions to identify the final states with leading signatures. We work out explicit predictions for the scalar leptoquark $S_{3}$; the flavor aspects of the analysis are analogous to those of the vector ones. We estimate improvements in mass reach for possible future $p p$ machines operating at center-of-mass energies $\sqrt{s}=33 \mathrm{TeV}$ (HE-LHC) and $100 \mathrm{TeV}$ (FCC-hh) [24,30]. For related recent works on leptoquark production and $R_{K, K^{*}}$, see [31-34]. 
The paper is organized as follows: In Sec. II, we review the requirements and constraints from flavor physics on the leptoquark's mass and couplings. Leptoquark branching ratios and single as well as pair production are discussed in Sec. III. Expectations for the flavor patterns of the leptoquark couplings are given in Sec. IV, together with corresponding branching ratios and signal strengths. In Sec. V, we conclude.

\section{THE SCALAR LEPTOQUARK $S_{3}$}

We denote by $S_{3}$ the scalar leptoquark that resides in the $(\overline{3}, 3,1 / 3)$ representation of the standard model (SM) gauge group. Its couplings to the SM fermions are given by the following Lagrangian:

$$
\begin{aligned}
\mathcal{L}_{\text {Yuk }}= & \lambda \bar{Q}_{L}^{C \alpha}\left(i \sigma^{2}\right)^{\alpha \beta}\left(S_{3}\right)^{\beta \gamma} L_{L}^{\gamma}+Y_{\kappa} \bar{Q}_{L}^{C \alpha}\left(i \sigma^{2}\right)^{\alpha \beta}\left(S_{3}^{\dagger}\right)^{\beta \gamma} Q_{L}^{\gamma} \\
& + \text { H.c., }
\end{aligned}
$$

where $\sigma^{2}$ is the second Pauli matrix and $\alpha, \beta$, and $\gamma$ are $S U(2)_{L}$ indices, whereas $\psi^{C}$ denotes the charge-conjugated spinor. We concentrate in this work on the first term that involves the coupling to leptons and quarks and assume the existence of a mechanism that forbids the second term that is potentially dangerous with regards to proton decay. Our interest is therefore focused on the Yukawa coupling matrix $\lambda$, a $3 \times 3$ matrix in flavor space with rows (columns) carrying a quark (lepton) flavor index that we suppress for the moment to avoid clutter. The $S_{3}$ can be represented in terms of its isospin components as

$$
S_{3}=\left(\begin{array}{cc}
S_{3}^{1 / 3} & \sqrt{2} S_{3}^{4 / 3} \\
\sqrt{2} S_{3}^{-2 / 3} & -S_{3}^{1 / 3}
\end{array}\right),
$$

where the superscripts denote the electric charge in units of $e$. The normalization is fixed to yield canonically normalized kinetic terms for the complex scalar components.

Expanding the Lagrangian (1) in terms of the isospin components, we obtain

$$
\begin{aligned}
\mathcal{L}_{\mathrm{QL}}= & -\sqrt{2} \lambda \bar{d}_{L}^{C} \ell_{L} S_{3}^{4 / 3}-\lambda \bar{d}_{L}^{C} \nu_{L} S_{3}^{1 / 3}+\sqrt{2} \lambda \bar{u}_{L}^{C} \nu_{L} S_{3}^{-2 / 3} \\
& -\lambda \bar{u}_{L}^{C} \ell_{L} S_{3}^{1 / 3}+\text { H.c. }
\end{aligned}
$$

The kinetic term for the leptoquark multiplet is written as

$$
\mathcal{L}_{\text {kin }}=\frac{1}{2} \operatorname{Tr}\left[\left(D_{\mu} S_{3}\right)^{\dagger} D^{\mu} S_{3}\right] .
$$

We assume the approximate mass degeneracy of the components within the multiplet. For the collider study in Sec. III, we implement the models (3) and (4) in Feynrules [35] to obtain the corresponding universal Feynrules output (UFO) [36]. The latter is used as input to the MadGraph event generator code [37].
To successfully accommodate present $R_{K^{(*)}}$ data with the $S_{3}$, one requires [21]

$$
\lambda_{b \mu} \lambda_{s \mu}^{*}-\lambda_{b e} \lambda_{s e}^{*} \simeq 1.1 \frac{M_{S_{3}}^{2}}{(35 \mathrm{TeV})^{2}} .
$$

Here, we label the element of the leptoquark Yukawa matrix $\lambda=\lambda_{q \ell}$ by the quark and lepton flavors to which it couples. By $S U(2)_{L}, \lambda_{U_{i} \ell}=V_{j i}^{*} \lambda_{D_{j} \ell}$, where $V$ denotes the Cabibbo Kobayashi Maskawa (CKM) matrix, and $U=u$, $c, t, D=d, s, b$ and $i, j=1,2,3$. Assuming (i) that the SM hierarchies for the quark Yukawas are intact in the leptoquark ones, couplings to third generation quarks are dominant $[7,38]$,

$\lambda_{d \ell} \sim\left(\epsilon^{3} \ldots \epsilon^{4}\right) \lambda_{b \ell}, \quad \lambda_{s \ell} \sim \epsilon^{2} \lambda_{b \ell}, \quad \ell=e, \mu, \tau$.

This can, for instance, be realized with a Froggatt-NielsenMechanism [39], where $\epsilon \sim 0.2$ denotes a flavor parameter of the size of the sine of the Cabibbo angle. The $\sim$ symbol indicates that a relation holds up to factors on the order of one. Charged lepton mass hierarchies are taken care of by the $S U(2)_{L}$ singlet leptons, i.e., the lepton doublets are neutral under the Froggatt-Nielsen symmetry, and no further suppressions in $\lambda_{q \ell}$ appear. Taking in addition into account that (ii) the beyond the standard model (BSM) effects in $R_{K, K^{*}}$ are predominantly from muons as opposed to electrons as corresponding contributions are consistent with those from global fits to the $b \rightarrow s \mu^{+} \mu^{-}$observables [40], a viable "simplified" benchmark $\lambda_{s}$ is obtained as

$$
\lambda_{s} \sim \lambda_{0}\left(\begin{array}{ccc}
0 & 0 & 0 \\
* & \epsilon^{2} & * \\
* & 1 & *
\end{array}\right) .
$$

Here, the entries denoted by " 0 " are of higher order in $\epsilon$; they are constrained by $\mu-e$ conversion and rare kaon decays and of no concern to the present analysis. The entries labeled with an asterisk are not needed to explain $|\Delta b|=|\Delta s|=1$ data. Equation (5) implies $\lambda_{0} \simeq M_{S_{3}} / 6.7 \mathrm{TeV}$. Allowing for order one factors in $\lambda_{s \mu}$, taken here to be between $1 / 3$ and 3 , one obtains the range

$$
M_{S_{3}} / 11.6 \mathrm{TeV} \lesssim \lambda_{0} \lesssim M_{S_{3}} / 3.9 \mathrm{TeV} .
$$

The parameter space (8) is well within the LHC limits on Drell-Yan production, to which $t$-channel leptoquarks contribute at tree level. Specifically, the Wilson coefficient $C_{b_{L} L}=v^{2} \lambda_{0}^{2} /\left(2 M_{S_{3}}^{2}\right)$ satisfies in our case $C_{b_{L} L} \lesssim 2 \times 10^{-3}$, where $v=246 \mathrm{GeV}$ denotes the vacuum expectation value (vev) of the Higgs, whereas experimentally, it is constrained only at the level of $10^{-2}$ for both electrons and muons [41]. Note that the effective theory is constructed to hold for leptoquark masses greater than the dilepton invariant mass, presently up to a few $\mathrm{TeV}$. However, also 
for smaller masses, effective theory bounds provide a useful approximation [42].

\section{COLLIDER SIGNATURES}

We discuss leptoquark decays and single leptoquark production at proton-proton colliders in Secs. III A and III B, respectively. In Sec. III C, we consider signatures with tops and jets. We occasionally use the symbol $\phi$ for a generic leptoquark.

\section{A. Decay and width}

Neglecting the masses of the decay products, the partial decay width of a scalar leptoquark $S_{3}$ with mass $M$ decaying to a lepton $\ell$ and a quark $q$ reads

$$
\Gamma\left(S_{3} \rightarrow q \ell\right)=c \frac{\left|\lambda_{q \ell}\right|^{2}}{16 \pi} M,
$$

where $c=2$ for $S_{3}^{4 / 3}, S_{3}^{-2 / 3}$ and $c=1$ for $S_{3}^{1 / 3}$; see Eq. (3). $\Gamma$ approximates the total width if the coupling $\lambda_{q \ell}$ is the dominant one. Note that the multibody decays induced by intermultiplet cascades such as $S_{3}^{-4 / 3} \rightarrow S_{3}^{-1 / 3} W^{-} \rightarrow b \nu W^{-}$ can become sizable for large masses. With couplings to the first and second quark generation being flavor suppressed, as, for instance, made explicit in (7) and the following text, the leptoquark predominantly decays to third generation quarks. The relevant parameter space in mass and leading coupling $\lambda_{0} \equiv \lambda_{b \mu}$ is illustrated in Fig. 1 for the $S_{3}^{4 / 3}$. The yellow (shaded) region corresponds to a narrow width, $\Gamma / M_{S_{3}} \lesssim 5 \%$, which translates to $\lambda_{0} \lesssim 1.1$. [Note that $\Gamma / M_{S_{3}} \lesssim 1 \%(10 \%)$ corresponds to $\lambda_{0} \lesssim 0.5(1.6)$.] The red band denotes the region that explains lepton nonuniversality (LNU) data, Eq. (8). In the hatched region above

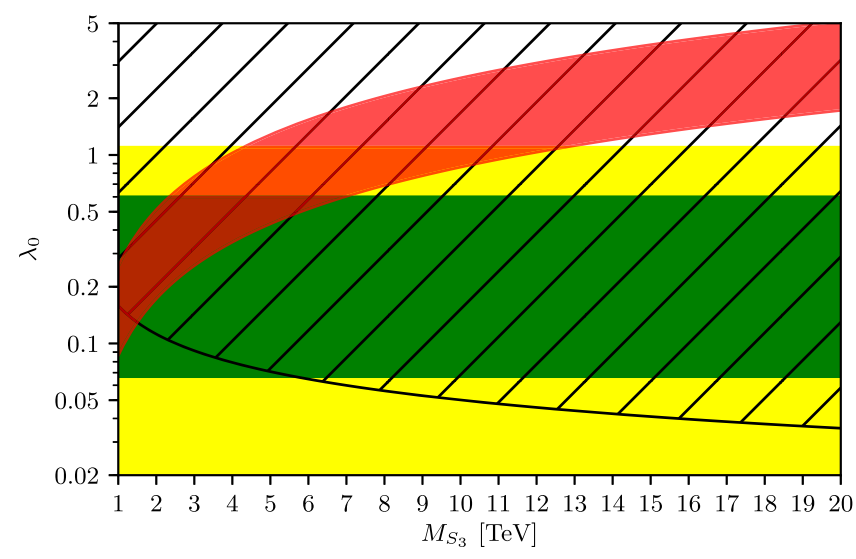

FIG. 1. Leptoquark parameter space, mass $M_{S_{3}}$ versus coupling, in the simplified scenario assuming a single dominant coupling $\lambda_{0}$ for $S_{3}^{4 / 3}$. The red band shows the range relevant to $R_{K}$, $R_{K^{*}}$ data (8), the yellow (shaded) region refers to a narrow width $\Gamma / M_{S_{3}} \lesssim 5 \%$, and in the hatched area above the black curve, $\Gamma>\Lambda_{\mathrm{QCD}}$. Flavor model predictions [16] are given by the green horizontal band. See text for details. the black curve, the leptoquark decays too rapid to form bound states, $\Gamma>\Lambda_{\mathrm{QCD}}$. Predictions from viable flavor models $\lambda_{b \mu} \sim c_{\ell}$, where $c_{\ell}$ is on the order of $\epsilon$ [16] (green horizontal band) are also shown. $B_{s}$-mixing data together with $R_{K}, R_{K^{*}}$ provide a data-driven upper limit on the mass of the $S_{3}$ leptoquark of $40 \mathrm{TeV}$. For such large masses, the coupling required by $B$-physics data becomes order one and approaches the perturbativity limit. In addition, the region of narrow width is left. Upper mass limits on the (gauge-like) vector leptoquarks are $45 \mathrm{TeV}$ and $20 \mathrm{TeV}$ for $V_{1}$ and $V_{3}$, respectively [21]. ${ }^{1}$

We list the dominant decays modes for the three leptoquark representations that can explain current LNU data [21] for the scalar isospin triplet:

$$
\begin{aligned}
& S_{3}^{+2 / 3} \rightarrow t \nu, \\
& S_{3}^{-1 / 3} \rightarrow b \nu, t \mu^{-}, \\
& S_{3}^{-4 / 3} \rightarrow b \mu^{-},
\end{aligned}
$$

where the vector isospin singlet decays as

$$
V_{1}^{+2 / 3} \rightarrow b \mu^{+}, t \nu
$$

and the vector isospin decays as

$$
\begin{aligned}
V_{3}^{-1 / 3} & \rightarrow b \nu, \\
V_{3}^{+2 / 3} & \rightarrow b \mu^{+}, t \nu, \\
V_{3}^{+5 / 3} & \rightarrow t \mu^{+} .
\end{aligned}
$$

As, for instance, $V_{1}^{-2 / 3} \rightarrow \bar{b} \mu^{-}$and $S_{3}^{-4 / 3} \rightarrow b \mu^{-}$lead both to a negatively charged lepton, tagging of the $b$-charge would be useful to identify the leptoquark type and its electric charge.

Note that some leptoquarks can undergo more than one decay into the third generation quarks, such as $S_{3}^{-1 / 3}$, Eq. (10), and in this case, by $S U(2)_{L}, \mathcal{B}\left(\phi \rightarrow b \nu_{\ell}\right) \sim$ $\mathcal{B}(\phi \rightarrow t \ell) \simeq 1 / 2$. Similarly, for $V_{1}$ (11) and $V_{3}$ (12), $\mathcal{B}(\phi \rightarrow b \ell) \sim \mathcal{B}\left(\phi \rightarrow t \nu_{\ell}\right) \simeq 1 / 2$.

\section{B. Single leptoquark production}

In Fig. 2, we show the leading order diagrams inducing single leptoquark production, followed by its decay. The production is in association with a lepton. The cross section is sensitive to the flavor coupling $\lambda_{q \ell}$. With the couplings to the first and second quark generations being flavor suppressed, the parton level production of the leptoquark is dominated by the third generation coupling. This continues to be the case at the hadron level, which can be inferred from Fig. 3. Therein, the triplet of (thin) curves corresponds

\footnotetext{
${ }^{1}$ Recent analysis of $B_{s}$ mixing [43] suggests lower upper limits on the leptoquark masses.
} 


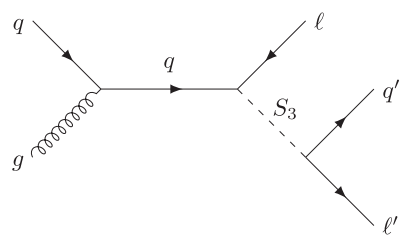

(a)

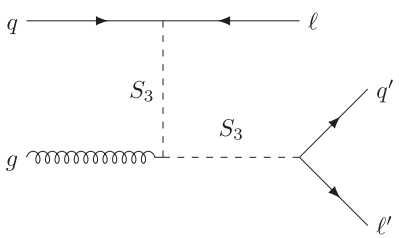

(b)

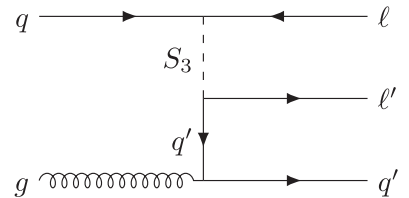

(c)

FIG. 2. Leading order diagrams for single leptoquark production and decay: Diagrams (a) and (b) correspond to resonant amplitudes. Diagram (c) corresponds to a nonresonant contribution, the effects of which are suppressed through kinematic cuts; see Sec. III B for details.
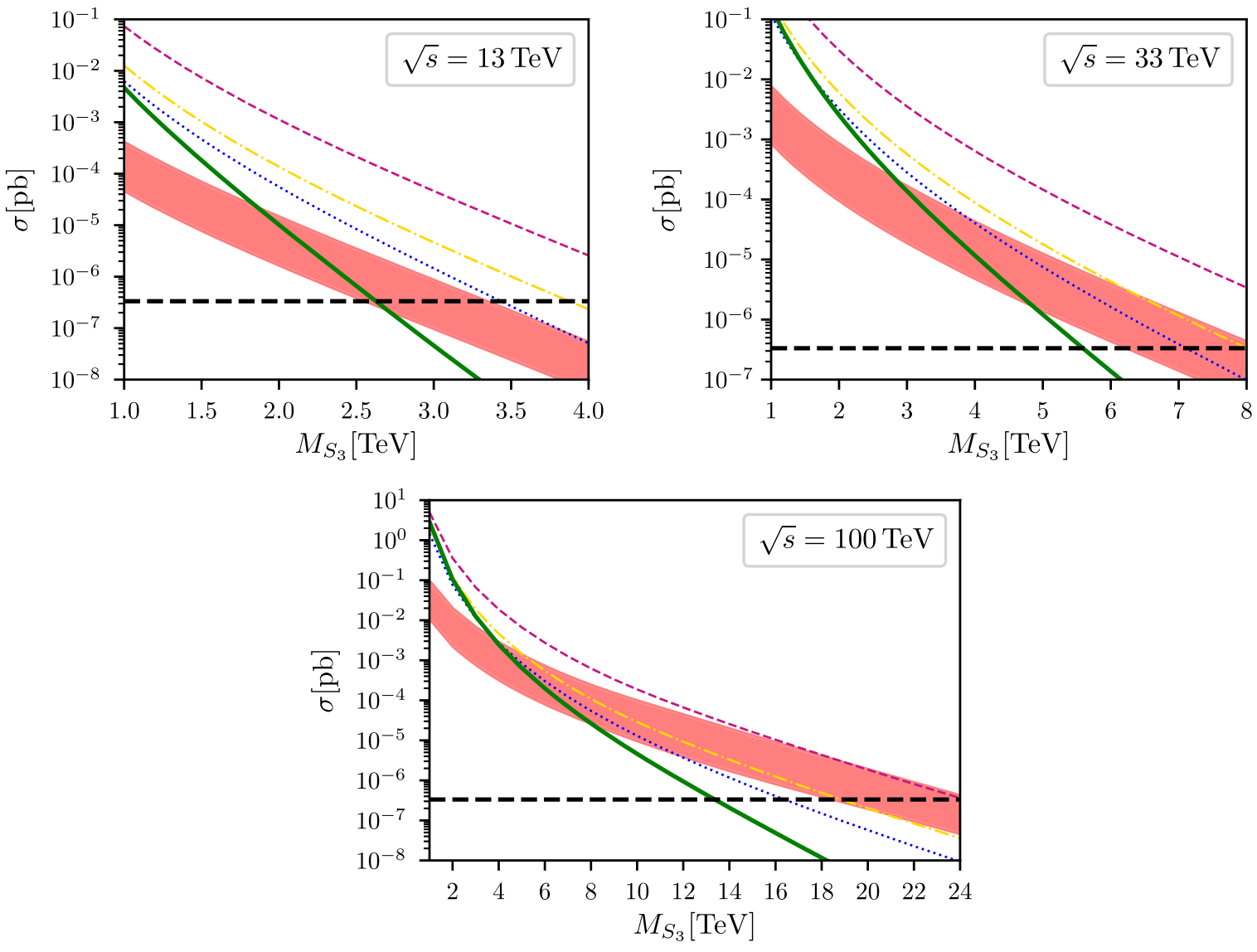

FIG. 3. Single leptoquark production cross section $\sigma\left(p p \rightarrow S_{3}^{-4 / 3} \mu^{+}+S_{3}^{+4 / 3} \mu^{-}\right)$as a function of the mass $M_{S_{3}}$ for $\sqrt{s}=13,33$, and $100 \mathrm{TeV}$. The red band corresponds to the flavor pattern (7) with $\lambda_{0}$ in accord with the $B$-anomalies (8). The triplet of (thin) curves illustrates the single production cross section with one coupling switched on at a time (from top to bottom: dashed pink, dashed-dotted orange, and dotted blue for $\lambda_{d \mu, s \mu, b \mu}$ set to one, respectively). The pair production cross section $\sigma\left(p p \rightarrow S_{3}^{-4 / 3} S_{3}^{+4 / 3}\right)$ is shown by the green (thick, solid) curve. The black (dashed) line corresponds to the absolute lower limit of the cross section below which one cannot produce a single event with integrated luminosity of $3000 \mathrm{fb}^{-1}$. See text for details.

to single production with a Yukawa coupling to only one down-type flavor switched on at a time (from top to bottom: dashed pink, dashed-dotted orange, and dotted blue for $\lambda_{d \mu, s \mu, b \mu}$ set to one, respectively). The ratio of $\lambda_{b \mu}$-induced to $\lambda_{x \mu}$-induced cross sections, $r_{x}, x=d, s$, gives the parton distribution function (PDF) suppression of $b$-production versus light flavor production. We find that $r_{s}$ is about a factor of a (few $)^{-1}$, and $r_{d}$ is on the order of $10^{-1}$ to $10^{-2}$, which are weaker than the respective flavor suppressions, Eq. (6), so indeed, beauty wins. In Fig. 3, we added the cross sections of $C P$-conjugated final states; in the absence of $C P$ violation, which is the limit we are working in, this amounts to a factor 2 in the single production cross section from valence quark-gluon fusion. Also shown in the two 


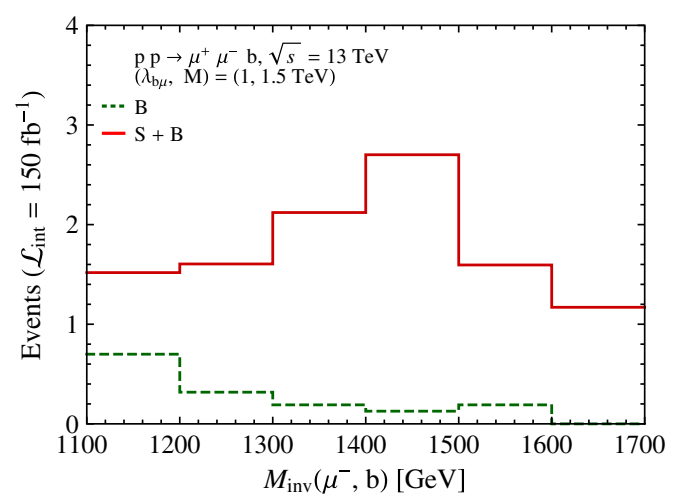

(a)

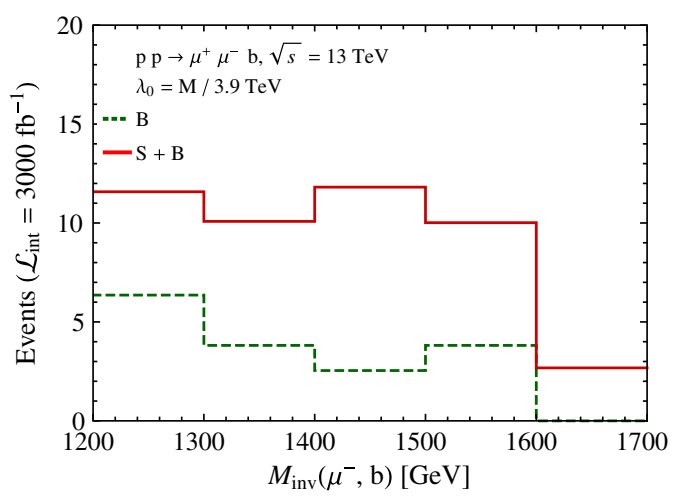

(b)

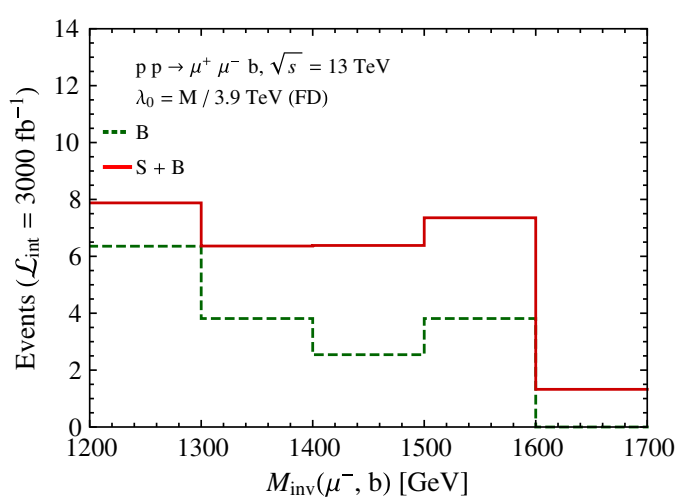

(c)

FIG. 4. Distribution of events over the invariant mass of the $\mu^{-}-b$-jet system from $p p \rightarrow b \mu^{+} \mu^{-}$and $p p \rightarrow \bar{b} \mu^{+} \mu^{-}$at $\sqrt{s}=13 \mathrm{TeV}$. All three plots correspond to a leptoquark with a mass of $1.5 \mathrm{TeV}$ : (a) corresponds to $\lambda_{b \mu}=1$ and (b) to the flavor pattern (7) with $\lambda_{0}=M / 3.9 \mathrm{TeV}$ in accord with $B$-anomalies (8). The pattern (15) on which (c) is based similarly addresses $B$-data but allows additionally for decays to taus. The dashed green (solid red) line represents background (signal and background) events. Kinematic cuts are described in the text.

additional plots are predictions for future proton-proton machines, a $33 \mathrm{TeV}$ HE-LHC and a $100 \mathrm{TeV}$ collider.

The corresponding numerical calculations are performed using MADGRAPH, version 2.6 [37] at leading order in quantum chromodynamics (QCD). We find that the largest uncertainties originate from the PDFs (we use LHAPDF [44]). For the single production (red band) linked to $R_{K^{(*)}}$ data (8), they grow from an order of $10 \%$ for $M \sim 1 \mathrm{TeV}$ to $\sim 35-40 \%$ for smaller cross sections of a few $\times 10^{-7} \mathrm{pb}$. The scale uncertainty-in our estimate, both the factorization and the renormalization scale are equal to half of the sum of the transverse masses of the final state particlesreaches $\sim 25 \%$.

In Fig. 3, the cross section for pair production $\sigma(p p \rightarrow$ $S_{3}^{-4 / 3} S_{3}^{+4 / 3}$ ) is shown by the solid green curve. We find, using MADGRAPH at leading order, that both PDF and scale uncertainties can reach $\mathcal{O}(40)$ percent towards $\sigma \sim$ a few $\times 10^{-7} \mathrm{pb}$. While the scale uncertainty is essentially flat, the PDF uncertainty drops to an order of $10 \%$ for lighter leptoquarks near a TeV. In the simplified benchmark (7), the $S_{3}^{ \pm 4 / 3}$ decays into $b \mu$, see (10), producing a $p p \rightarrow b b \mu \mu$ signature. Pair production of another component of the $S_{3}$ can give $t t \mu \mu, b b E_{\text {miss }}, b t \mu E_{\text {miss }}$, or $t t E_{\text {miss }}$ final states.

For low masses, pair production has a cross section larger than that of single production (red band) linked to $R_{K^{(*)}}$ data (8), while the single production cross section is larger for higher masses [45]. Naively about a factor $\sim 2(5)$ in mass reach can be gained in pair production at a $33(100) \mathrm{TeV}$ collider relative to $13 \mathrm{TeV}$ and for comparable luminosity of $3000 \mathrm{fb}^{-1}$. The potential gain for single production is somewhat larger: about a factor 2.5 (7) in the target parameter space, the red band, for 33(100) TeV. While this gives an idea about the accessible ranges, dedicated simulations are needed to estimate the reach more reliably.

We simulate events for a $1.5 \mathrm{TeV}$ leptoquark and different couplings at the $\sqrt{s}=13 \mathrm{TeV}$ LHC. In Fig. 4, we present the corresponding distributions for signal and background as a function of $M_{\text {inv }}\left(\mu^{-}, b\right)$, the invariant mass of the $\mu^{-}-b$ system. To enhance the significance and to study a situation where the $b$-charge is not tagged, we add the $C P$-conjugate process $p p \rightarrow \bar{b} \mu^{+} \mu^{-}$in both signal and background. The corresponding calculations are performed at leading order in QCD using MADGRAPH, version 2.6 [37] 
for the event generation, PYTHIA [46] for the parton showering and hadronization and DELPHES 3 [47] for the fast detector simulation. For the muon isolation we, follow [28], whereas all the other criteria are taken from the default DELPHES card for the CMS detector. To account for the QCD corrections at NLO, we multiply signals and backgrounds with corresponding global k-factors. For the signal, we use $k \sim 1.5$ [48], whereas for the background, we use the value that we obtain by comparing the NLO and LO calculations at fixed order in MADGRAPH. For the analysis of the signal and background we use MADANALYsis 5 [49].

For these evaluations, we adopt the following kinematic cuts: We accept events containing two opposite-charge muons and a $b$-jet and require for the transverse momenta and absolute pseudorapidities of each of these final states to exceed $45 \mathrm{GeV}$ and to be smaller than 2.1 , respectively. Furthermore, for the angular separation between muon and a $b$-jet, we have $\Delta R>0.3$, and also we cut on the invariant mass of the opposite-charge muon-pair $M_{\text {inv }}>110 \mathrm{GeV}$. For the final event selection, we adopt the cut on the scalar sum of the transverse momenta of the muon pair and leading $b$-jet $S_{T}\left(\mu_{1}, \mu_{2}, b_{1}\right)>250 \mathrm{GeV}$ [28]. We use $M_{\text {inv }}(\mu, b$-jet $)$ as a discriminating variable between signal and background and adjust the corresponding cut to a given mass and coupling hypothesis in order to maximize the significance of the signal.

The approximate expected discovery significance for the first case (a) $\lambda_{b \mu}=1$, which is a priori unrelated to LNU data, is around $4 \sigma$ for an integrated luminosity of $150 \mathrm{fb}^{-1}$. In the second case (b) where $\lambda_{b \mu}$ saturates the upper limit in (7) and (8), we find around $5 \sigma$ at $3000 \mathrm{fb}^{-1}$. The significance for the third case (c) is smaller, somewhat below $3 \sigma$ due to the twice smaller branching fraction into $b \mu$; see (15). To compute these significances, we used the approximate formula from [50] and took into account both $\mu^{-}-b$ and $\mu^{+}-\bar{b}$ signals in the data sample.

\section{Tops and jets}

We briefly discuss single production into top and jet plus dilepton final states, which complements searches into $b$-quarks.

The processes $g b \rightarrow \mu^{+} \phi\left(\rightarrow b \mu^{-}\right)$are related to $g b \rightarrow$ $\nu \phi\left(\rightarrow t \mu^{-}\right)$by $S U(2)_{L}$ and arise at the same order of flavor counting. We recall the factor $1 / 2$ in the leading $S_{3}^{-1 / 3}$ branching ratios as within our approximations, the leptoquark decays to both $b \nu$ and $t \ell$ via $\lambda_{0}$ at equal rate. However, the $t \mu \nu$ final state has larger background because SM processes from $W \rightarrow \ell \nu$ cannot be removed equally well as $Z \rightarrow \ell \ell$. On the other hand, if the flavor suppression of the second generation quark coupling is not realized in nature, $t \mu \mu$ final states, induced by $\lambda_{c \mu} \simeq \lambda_{s \mu}$, could potentially be interesting. For $\lambda_{b \mu}=\lambda_{s \mu}$, one finds for the branching ratio $\mathcal{B}(\phi \rightarrow t \mu) \simeq 1 / 4$; however, the leptoquark coupling drops by an order of magnitude, $\lambda_{0}=0.03 \mathrm{M} / \mathrm{TeV}$, as dictated by
(5). Despite the PDF enhancement from charm relative to $b$, this democratic scenario results in about two orders of magnitude smaller cross sections relative to $p p \rightarrow b \mu \mu$.

Inverted hierarchies $\lambda_{s \mu} \gg \lambda_{b \mu}$ are in conflict with flavor symmetry (see Sec. IV) but not excluded experimentally in the simplified scenario with two entries only (7). This extreme scenario, however, does not improve the situation regarding $t \mu \mu$, as the branching ratio into $t \mu$ is suppressed as $\left|\lambda_{b \mu} / \lambda_{s \mu}\right|^{2}$, while the product of couplings is fixed by $B$-data (5). On the other hand, jet plus dileptons benefits from the large second generation Yukawa while having an order one branching ratio. Note that jet plus charged lepton final states can arise from several components of the $S U(2)_{L}$ multiplets (10)-(12). Using the upper limit $\lambda_{s \mu} \lesssim$ $M / 2 \mathrm{TeV}$ from Drell-Yan production at the LHC [41] and taking into account the PDF enhancement, second quark generation cross sections $\sigma\left(p p \rightarrow\left(j \mu^{-}\right) \mu^{+}+\left(j \mu^{+}\right) \mu^{-}\right)$ can be about an order of magnitude larger than those of the maximum third generation ones, in accord with $B$-anomalies (8), shown by the red band in Fig. 3. A detailed analysis of the sensitivity to inverted hierarchies including reconstruction efficiencies is left for future work.

\section{FLAVOR BENCHMARKS}

We explain how the simplified pattern (7) can arise in models of flavor and give more general Yukawa patterns. We are, in particular, interested in theoretical predictions for the entries with an asterisk that potentially induce leptoquark signals with electrons or taus and LFV, which affects the collider phenomenology.

The most general approach, treating all entries as free parameters only constrained by upper limits and (5), presently does not suffice to identify the dominant collider signatures. We therefore suggest to study benchmarks. They are motivated by viable flavor symmetries that successfully explain SM flavor and consistency with data.

A simultaneous explanation of the LNU ratios $R_{K}, R_{K^{*}}$, and $B \rightarrow K^{*} \mu^{+} \mu^{-}$angular distributions is possible with BSM effects in couplings to muons alone. Hence, from this perspective, leptoquark couplings to electrons are not necessary. Lepton species' isolation patterns can be engineered

TABLE I. Branching fractions of leptoquark $S_{3}^{-4 / 3}$ decaying to $b \ell$ and $j \ell, \ell=e, \mu, \tau$ for different flavor benchmarks (13), (14), and (15); see text for details. Corresponding branching fractions of the $S_{3}^{-1 / 3}$ satisfy $\mathcal{B}\left(S_{3}^{-1 / 3} \rightarrow t \ell\right), \mathcal{B}\left(S_{3}^{-1 / 3} \rightarrow b \nu\right) \sim$ $\mathcal{B}\left(S_{3}^{-4 / 3} \rightarrow b \ell\right) / 2$ and for jets $\mathcal{B}\left(S_{3}^{-1 / 3} \rightarrow j \ell\right), \mathcal{B}\left(S_{3}^{-1 / 3} \rightarrow j \nu\right) \sim$ $\mathcal{B}\left(S_{3}^{-4 / 3} \rightarrow j \ell\right) / 2$.

\begin{tabular}{lcccccc}
\hline \hline & $b \mu$ & $b e$ & $b \tau$ & $j \mu$ & $j e$ & $j \tau$ \\
\hline$\lambda_{\mu}$ & 1 & $\delta^{2}$ & $\delta^{2}$ & $\epsilon^{4}$ & $\epsilon^{4} \delta^{2}$ & $\epsilon^{4} \delta^{2}$ \\
$\tilde{\lambda}_{\mu}$ & 1 & $\delta^{2}$ & $\delta^{2}$ & $\left(c_{\nu} \kappa / c_{\ell}\right)^{2}$ & $\left(c_{\nu} \kappa / c_{\ell}\right)^{2}$ & $\left(c_{\nu} \kappa / c_{\ell}\right)^{2}$ \\
$\lambda_{\mathrm{FD}}$ & $1 / 2$ & $\kappa_{e}^{2} / 2$ & $1 / 2$ & $\rho^{2} / 2$ & $\rho^{2} \kappa_{e}^{2} / 2$ & $\rho^{2} / 2$ \\
\hline \hline
\end{tabular}


TABLE II. Parametric signal strength of $p p \rightarrow b \ell \ell^{\prime}$ and $p p \rightarrow j \ell \ell^{\prime}$ final states from single leptoquark $S_{3}^{-4 / 3}$ production for different flavor benchmarks (13), (14), and (15); see text for details.

\begin{tabular}{lcccccccccccc}
\hline \hline & $b \mu \mu$ & $b e \mu$ & $b \tau \mu$ & $b e e$ & $b e \tau$ & $b \tau \tau$ & $j \mu \mu$ & $j e \mu$ & $j \tau \mu$ & $j e e$ & $j e \tau$ & $j \tau \tau$ \\
\hline$\lambda_{\mu}$ & $c_{\ell}^{2}$ & $c_{\ell}^{2} \delta^{2}$ & $c_{\ell}^{2} \delta^{2}$ & $c_{\ell}^{2} \delta^{4}$ & $c_{\ell}^{2} \delta^{4}$ & $c_{\ell}^{2} \delta^{4}$ & $c_{\ell}^{2} \epsilon^{4}$ & $c_{\ell}^{2} \delta^{2} \epsilon^{4}$ & $c_{\ell}^{2} \delta^{2} \epsilon^{4}$ & $c_{\ell}^{2} \delta^{4} \epsilon^{4}$ & $c_{\ell}^{2} \delta^{4} \epsilon^{4}$ & $c_{\ell}^{2} \delta^{4} \epsilon^{4}$ \\
$\tilde{\lambda}_{\mu}$ & $c_{\ell}^{2}$ & $c_{\ell}^{2} \delta^{2}$ & $c_{\ell}^{2} \delta^{2}$ & $c_{\ell}^{2} \delta^{4}$ & $c_{\ell}^{2} \delta^{4}$ & $c_{\ell}^{2} \delta^{4}$ & $\left(c_{\nu} \kappa\right)^{2}$ & $\left(c_{\nu} \kappa\right)^{2}$ & $\left(c_{\nu} \kappa\right)^{2}$ & $\left(c_{\nu} \kappa \delta\right)^{2}$ & $\left(c_{\nu} \kappa \delta\right)^{2}$ & $\left(c_{\nu} \kappa \delta\right)^{2}$ \\
$\lambda_{\mathrm{FD}}$ & $\lambda_{0}^{2} / 2$ & $\lambda_{0}^{2} \kappa_{e}^{2} / 2$ & $\lambda_{0}^{2} / 2$ & $\lambda_{0}^{2} \kappa_{e}^{4} / 2$ & $\lambda_{0}^{2} \kappa_{e}^{2} / 2$ & $\lambda_{0}^{2} / 2$ & $\lambda_{0}^{2} \rho^{2} / 2$ & $\lambda_{0}^{2} \rho^{2} \kappa_{e}^{2} / 2$ & $\lambda_{0}^{2} \rho^{2} / 2$ & $\lambda_{0}^{2} \rho^{2} \kappa_{e}^{4} / 2$ & $\lambda_{0}^{2} \rho^{2} \kappa_{e}^{2} / 2$ & $\lambda_{0}^{2} \rho^{2} / 2$ \\
\hline \hline
\end{tabular}

with discrete, non-abelian flavor symmetries such as $A_{4}$ [7]. For second generation leptons, these read

$$
\lambda_{\mu} \sim c_{\ell}\left(\begin{array}{ccc}
0 & \epsilon^{4} & 0 \\
0 & \epsilon^{2} & 0 \\
0 & 1 & 0
\end{array}\right) \rightarrow c_{\ell}\left(\begin{array}{ccc}
\delta \epsilon^{4} & \epsilon^{4} & \delta \epsilon^{4} \\
\delta \epsilon^{2} & \epsilon^{2} & \delta \epsilon^{2} \\
\delta & 1 & \delta
\end{array}\right),
$$

or one that avoids the CKM suppression for the second generation quarks [16],

$$
\begin{aligned}
& \tilde{\lambda}_{\mu} \sim\left(\begin{array}{ccc}
0 & c_{\ell} \epsilon^{4} & 0 \\
c_{\nu} \kappa & c_{\nu} \kappa & c_{\nu} \kappa \\
0 & c_{\ell} & 0
\end{array}\right) \\
& \rightarrow\left(\begin{array}{ccc}
c_{\nu} \kappa \epsilon^{2} & c_{\ell} \epsilon^{4}+c_{\nu} \kappa \epsilon^{2} & c_{\nu} \kappa \epsilon^{2} \\
c_{\nu} \kappa & c_{\ell} \epsilon^{2}+c_{\nu} \kappa & c_{\nu} \kappa \\
c_{\ell} \delta+c_{\nu} \kappa \epsilon^{2} & c_{\ell} & c_{\ell} \delta+c_{\nu} \kappa \epsilon^{2}
\end{array}\right) .
\end{aligned}
$$

In Eqs. (13) and (14), all vevs $c_{\ell}, c_{\nu}$, and $\kappa$ are on the order of $\epsilon^{n}, n \geq 1$. $\delta$ is a small parameter of second order in the vevs; see [16] for details.

Patterns (13) and (14) receive corrections from rotating flavor to mass basis and from higher order spurion insertions [16], both of which are incorporated in the matrices to the right of the arrow. As a result, in addition to those to muons, leptoquarks couple to all leptons and third generation quarks, and LFV arises.

For the third benchmark, we employ a more general parametrization and impose experimental constraints [7] "flavor data":

$$
\lambda_{\mathrm{FD}}=\lambda_{0}\left(\begin{array}{ccc}
\rho_{d} \kappa_{e} & \rho_{d} & \rho_{d} \kappa_{\tau} \\
\rho \kappa_{e} & \rho & \rho \kappa_{\tau} \\
\kappa_{e} & 1 & \kappa_{\tau}
\end{array}\right), \quad \kappa_{\tau} \sim 1
$$

Here, we allow for quark flavor suppressions $\rho_{d}=\lambda_{d \ell} / \lambda_{b \ell}$ and $\rho=\lambda_{s \ell} / \lambda_{b \ell}$, with larger couplings for higher generations, in concordance with the observed quark mass pattern. In addition, a suppression factor $\kappa_{e}$ for the electrons is accounted for. The phenomenologically viable range for $\lambda_{\mathrm{FD}}$ parameters in Eq. (15) is [7]

$$
\begin{aligned}
\rho_{d} & \lesssim 0.02, \quad \kappa_{e} & \lesssim 0.5, \quad 10^{-4} \lesssim \rho \lesssim 1, \\
\kappa_{e} / \rho & \lesssim 0.5, \quad \rho_{d} / \rho & \lesssim 1.6 .
\end{aligned}
$$

The MEG experiment [51] can put a limit on $\kappa_{e} / \rho$ at the level of 0.2 in the future [7].

Branching fractions of $S_{3}$ to $b \ell$ and $j \ell, \ell=e, \mu, \tau$ in the three benchmarks are presented in Table I. They are also useful to estimate signatures in leptoquark pair production. Predictions for the vector leptoquarks follow analogous flavor patterns: Modulo the slightly different vevs [16] in $\tilde{\lambda}_{\mu}$, one obtains in addition $\mathcal{B}\left(V_{1} \rightarrow b \ell\right) \sim \mathcal{B}\left(S_{3}^{-4 / 3} \rightarrow b \ell\right) / 2$ for similar masses. In Table II, we give the parametric signal strength for single leptoquark production using the narrow-width approximation,

$$
\sigma(p p \rightarrow \phi(\rightarrow q \ell) \ell)=\sigma(p p \rightarrow \phi \ell) \mathcal{B}(\phi \rightarrow q \ell),
$$

in the benchmarks for different final state flavors. In both Tables I and II, we give the leading terms in the vev expansion, $c_{\ell}, c_{\nu}, \kappa<1$, and the flavor factors $\rho, \rho_{d}, \kappa_{e}<1$.

Hierarchies in $\lambda_{\mu}$ and $\tilde{\lambda}_{\mu}$ are identical for all $b$-final states, but the jet ${ }^{2}$ signals are less suppressed in $\tilde{\lambda}_{\mu}$. bee and jee channels are strongly suppressed in both benchmarks (13) and (14). For $\lambda_{\mathrm{FD}}$, the situation depends on how strong flavor suppressions are. A small $\rho$ implies a suppressed $\kappa_{e}$, (16). We identify two limits:

(A) $\rho, \kappa_{e}$ are order one, then either $\lambda_{0}$ has to be small, or, if $\lambda_{0}$ is order one, as well, then leptoquark masses are in the multi-10 $\mathrm{TeV}$ range. In either case, there is no leptoquark-induced $b \mu \mu$ signal at the LHC.

(B) $\rho, \kappa_{e} \ll 1$, then $\lambda_{0}$ is sizable, while leptoquark masses can be $\mathrm{TeV}$-ish, and the jet and electron modes are suppressed.

Case (B) resembles the situation for benchmarks $\lambda_{\mu}$ and $\tilde{\lambda}_{\mu}$. Constraints on $\kappa_{e}$ are therefore important to control final states with electrons. For $\kappa_{e} \ll 1$, the $e e$ or $e \mu$ modes would be SM-like. $\kappa_{e}$ can be constrained from $b \rightarrow$ see or $b \rightarrow$ $s e \mu$ processes together with $b \rightarrow s \mu \mu$. Due to reduced uncertainties, angular observables in $B \rightarrow K^{*}(\rightarrow K \pi) e e$ decays are promising [17].

\footnotetext{
${ }^{2}$ We use "jet" for an object from gluons, $u, d, s$ and $c$-quarks and antiquarks, as opposed to a $b$-jet, made out of $b$ and $\bar{b}$.
} 
In the presence of sizable couplings to more than one lepton species, such as muons and taus, there are two main aspects to single production: First, the signal in $p p \rightarrow$ $(\phi \rightarrow b \mu) \mu$ drops because the leptoquark also has a decay rate into $b \tau$. This happens in plot (c) of Fig. 4. Second, LFV arises, such as $p p \rightarrow(\phi \rightarrow b \tau) \mu$ and $p p \rightarrow(\phi \rightarrow b \mu) \tau$. This can be searched for in a complementary way in $B \rightarrow K^{(*)} \tau \mu$ decays.

Up to cuts and detection efficiencies, $S_{3}$-induced $p p \rightarrow t \mu \mu$, which arises from charm quarks (see Fig. 2), is suppressed by $\epsilon^{4},\left(c_{\nu} \kappa / c_{\ell}\right)^{2}$, and $\rho^{2}$ in scenario $\lambda_{\mu}, \tilde{\lambda}_{\mu}$, and $\lambda_{\text {fd }}$, respectively, with respect to $p p \rightarrow b \mu \mu$. Using the same approximations, the $p p \rightarrow t \mu \nu$ and $p p \rightarrow b \nu \nu$ signal strengths are the same as that for $p p \rightarrow b \mu \mu$.

Both $S_{3}^{-4 / 3}$ and $S_{3}^{-1 / 3}$ produce $j \ell \ell^{(\prime)}$ final states (10); hence, the parametric signal strength of all jet modes in Table II is additionally enhanced by a factor $\sim 3 / 2$. Here, we used that the strange and charm PDFs are similar in size within our approximations.

\section{CONCLUSION}

The $\mathrm{TeV}$ mass leptoquarks can be singly produced at hadron colliders in association with a lepton. $B$-physics data, which hint at a BSM contribution in $b \rightarrow s \mu \mu$ processes, while one in $b \rightarrow$ see may be discarded by Occam's razor, together with flavor model building identify $p p \rightarrow \phi \mu \rightarrow$ $b \mu \mu$ and two modes with missing energy $p p \rightarrow \phi \nu \rightarrow b \nu \nu$ and $p p \rightarrow \phi \nu \rightarrow t \mu \nu$, as the channels with leading cross sections. While this highlights $b \mu \mu$ as a prime channel, signatures with further final states can also be sizable and should be explored. The reasons are, first, to advance our understanding of flavor by probing lepton and quark flavorspecific couplings in the leptoquarks' Yukawa matrix, as opposed to rare decays (5), which constrain products of couplings. Second, exploration of further single production modes serves as a cross check with other measurements, such as leptoquark pair production, and indirect searches, notably Drell-Yan production and semileptonic rare $b$-decays.

Due to the higher cross section for lower masses (see Fig. 3), we encourage searches for leptoquarks from pair production decaying to a $b$-quark and a lepton, or a top quark and a lepton, as in (10)-(12).
Semileptonic $b$-decays can be probed at LHCb and Belle II and allow access to lepton-specific couplings of all three generations, which could improve benchmarks (15) and aid collider searches. Corresponding processes are $b \rightarrow$ see and LFV, $b \rightarrow$ se $\mu$ and those into taus. Studies of the angular distribution in $B \rightarrow K^{*} e e$ similar to $B \rightarrow K^{*} \mu \mu$ [17,52] and searches for $B \rightarrow K^{(*)} e(\mu, \tau)$ and $B \rightarrow K^{(*)} \mu \tau$ at the level of $10^{-8}$ and lower and $B_{s} \rightarrow e \mu$ at $\mathcal{O}\left(10^{-11}\right)$ [7] should be pursued to obtain meaningful constraints on the leptoquark flavor matrix.

Discrimination between different quark flavors can be achieved by comparing signatures induced by the third generation quark coupling to the ones induced by first two generations, such as $b \ell \ell$ to $j \ell \ell$. Flavor symmetries predict the latter to be suppressed or at least not enhanced relative to the former, as a result of the corresponding quark hierarchies, e.g., see patterns (13), (14), and (15). An experimental search could put this prediction to a test. Evidence for an inverted quark hierarchy $\lambda_{s \ell} \gg \lambda_{b \ell}$ would suggest an origin of flavor outside of symmetries, such as anarchy. Corresponding final states from pair production are $j j \ell \ell^{\prime}$, where lepton species $\ell, \ell^{\prime}$ can be the same or different.

Our analysis shows that the LHC, even with $3 \mathrm{ab}^{-1}$, is not able to cover the full targeted parameter space (see Fig. 3), where expectations for future $p p$ colliders with higher center of mass energy and comparable luminosity are also shown. For measurements to be useful for flavor, $b$-identification is required. Additionally, the ability to tag the flavor of the $b$ would allow one to measure the leptoquark's electric charge and distinguish leptoquark representations.

\section{ACKNOWLEDGMENTS}

We are happy to thank Ulrik Egede, Yossi Nir, and José Zurita for useful discussions. This project is supported in part by the Bundesministerium für Bildung und Forschung (BMBF).

Note added.-During the finalization of this work a related study on leptoquark production at colliders appeared [53].
[1] G. Hiller and F. Krüger, Phys. Rev. D 69, 074020 (2004).

[2] R. Aaij et al. (LHCb Collaboration), Phys. Rev. Lett. 113, 151601 (2014).

[3] R. Aaij et al. (LHCb Collaboration), J. High Energy Phys. 08 (2017) 055.

[4] G. Hiller and M. Schmaltz, Phys. Rev. D 90, 054014 (2014).
[5] B. Gripaios, M. Nardecchia, and S. A. Renner, J. High Energy Phys. 05 (2015) 006.

[6] R. Barbieri, G. Isidori, A. Pattori, and F. Senia, Eur. Phys. J. C 76, 67 (2016).

[7] I. de Medeiros Varzielas and G. Hiller, J. High Energy Phys. 06 (2015) 072. 
[8] I. Doršner, S. Fajfer, A. Greljo, J. F. Kamenik, and N. Košnik, Phys. Rep. 641, 1 (2016).

[9] S. Fajfer and N. Košnik, Phys. Lett. B 755, 270 (2016).

[10] D. Bečirević, N. Košnik, O. Sumensari, and R. Zukanovich Funchal, J. High Energy Phys. 11 (2016) 035.

[11] R. Alonso, B. Grinstein, and J. Martin Camalich, J. High Energy Phys. 10 (2015) 184.

[12] L. Calibbi, A. Crivellin, and T. Ota, Phys. Rev. Lett. 115, 181801 (2015).

[13] S. Sahoo and R. Mohanta, Phys. Rev. D 91, 094019 (2015).

[14] D. Bečirević, S. Fajfer, and N. Košnik, Phys. Rev. D 92, 014016 (2015).

[15] P. Cox, A. Kusenko, O. Sumensari, and T. T. Yanagida, J. High Energy Phys. 03 (2017) 035.

[16] G. Hiller, D. Loose, and K. Schönwald, J. High Energy Phys. 12 (2016) 027.

[17] G. Hiller and M. Schmaltz, J. High Energy Phys. 02 (2015) 055.

[18] W. Altmannshofer, P. Stangl, and D. M. Straub, Phys. Rev. D 96, 055008 (2017).

[19] G. D’Amico, M. Nardecchia, P. Panci, F. Sannino, A. Strumia, R. Torre, and A. Urbano, J. High Energy Phys. 09 (2017) 010.

[20] B. Capdevila, A. Crivellin, S. Descotes-Genon, J. Matias, and J. Virto, J. High Energy Phys. 01 (2018) 093.

[21] G. Hiller and I. Nišandžić, Phys. Rev. D 96, 035003 (2017).

[22] L. S. Geng, B. Grinstein, S. Jäger, J. Martin Camalich, X. L. Ren, and R. X. Shi, Phys. Rev. D 96, 093006 (2017).

[23] M. Ciuchini, A. M. Coutinho, M. Fedele, E. Franco, A. Paul, L. Silvestrini, and M. Valli, Eur. Phys. J. C 77, 688 (2017).

[24] V. Shiltsev, arXiv:1705.02011.

[25] G. Aad et al. (ATLAS Collaboration), Eur. Phys. J. C 76, 5 (2016).

[26] M. Aaboud et al. (ATLAS Collaboration), New J. Phys. 18, 093016 (2016).

[27] V. Khachatryan et al. (CMS Collaboration), Phys. Rev. D 93, 032004 (2016).

[28] V. Khachatryan et al. (CMS Collaboration), Phys. Rev. D 93, 032005 (2016) [95, 039906(E) (2017)].

[29] B. Diaz, M. Schmaltz, and Y. M. Zhong, J. High Energy Phys. 10 (2017) 097.

[30] M. Mangano, CERN Yellow Report CERN Report No. 2017-003-M.

[31] I. Doršner, S. Fajfer, D. A. Faroughy, and N. Košnik, J. High Energy Phys. 10 (2017) 188.
[32] U. K. Dey, D. Kar, M. Mitra, M. Spannowsky, and A. C. Vincent, arXiv:1709.02009.

[33] D. Buttazzo, A. Greljo, G. Isidori, and D. Marzocca, J. High Energy Phys. 11 (2017) 044.

[34] B. C. Allanach, B. Gripaios, and T. You, arXiv:1710.06363.

[35] A. Alloul, N. D. Christensen, C. Degrande, C. Duhr, and B. Fuks, Comput. Phys. Commun. 185, 2250 (2014).

[36] C. Degrande, C. Duhr, B. Fuks, D. Grellscheid, O. Mattelaer, and T. Reiter, Comput. Phys. Commun. 183, 1201 (2012).

[37] J. Alwall, R. Frederix, S. Frixione, V. Hirschi, F. Maltoni, O. Mattelaer, H.-S. Shao, T. Stelzer, P. Torrielli, and M. Zaro, J. High Energy Phys. 07 (2014) 079.

[38] P. H. Chankowski, K. Kowalska, S. Lavignac, and S. Pokorski, Phys. Rev. D 71, 055004 (2005).

[39] C. D. Froggatt and H. B. Nielsen, Nucl. Phys. B147, 277 (1979).

[40] S. Descotes-Genon, L. Hofer, J. Matias, and J. Virto, J. High Energy Phys. 06 (2016) 092; T. Hurth, F. Mahmoudi, and S. Neshatpour, J. High Energy Phys. 12 (2014) 053; W. Altmannshofer and D. M. Straub, Eur. Phys. J. C 75, 382 (2015); F. Beaujean, C. Bobeth, and D. van Dyk, Eur. Phys. J. C 74, 2897 (2014) 74, 3179(E) (2014).

[41] A. Greljo and D. Marzocca, Eur. Phys. J. C 77, 548 (2017).

[42] A. Bessaa and S. Davidson, Eur. Phys. J. C 75, 97 (2015).

[43] L. Di Luzio, M. Kirk, and A. Lenz, arXiv:1712.06572.

[44] A. Buckley, J. Ferrando, S. Lloyd, K. Nordström, B. Page, M. Rüfenacht, M. Schönherr, and G. Watt, Eur. Phys. J. C 75, 132 (2015).

[45] I. Dorsner, S. Fajfer, and A. Greljo, J. High Energy Phys. 10 (2014) 154.

[46] T. Sjöstrand, S. Ask, J. R. Christiansen, R. Corke, N. Desai, P. Ilten, S. Mrenna, S. Prestel, C. O. Rasmussen, and P. Z. Skands, Comput. Phys. Commun. 191, 159 (2015).

[47] J. de Favereau, C. Delaere, P. Demin, A. Giammanco, V. Lemaître, A. Mertens, and M. Selvaggi (DELPHES 3 Collaboration), J. High Energy Phys. 02 (2014) 057.

[48] J. B. Hammett and D. A. Ross, J. High Energy Phys. 07 (2015) 148.

[49] E. Conte, B. Fuks, and G. Serret, Comput. Phys. Commun. 184, 222 (2013).

[50] G. Cowan, K. Cranmer, E. Gross, and O. Vitells, Eur. Phys. J. C 71, 1554 (2011) [73, 2501(E) (2013)].

[51] A. M. Baldini et al., arXiv:1301.7225.

[52] S. Wehle et al. (Belle Collaboration), Phys. Rev. Lett. 118, 111801 (2017).

[53] I. Doršner and A. Greljo, arXiv:1801.07641. 